\title{
Quantum properties of QCD string fragmentation
}

\author{
Šárka Todorova-Nová ${ }^{\text {,a }}$ \\ ${ }^{1}$ Institute of Particle and Nuclear Physics of Charles University, V Holešovičkách 2, 18000 Praha 8, Czech \\ Republic
}

\begin{abstract}
A simple quantization concept for a 3-dim QCD string is used to derive properties of QCD flux tube from the mass spectrum of light mesons and to predict observable quantum effects in correlations between adjacent hadrons. The quantized fragmentation model is presented and compared with experimental observations.
\end{abstract}

\section{Introduction}

The Lund string fragmentation[1], which is using a 1-dimensional string to model the QCD confinement, imposes a space-like distance between the string breakup vertices forming a hadron. The model has to rely on the concept of quantum tunneling to generate intrinsic transverse momenta of hadrons. The local charge and momentum conservation holds in the break-up vertex but there are no correlations, in the string transverse plane, between non-adjacent hadrons.

The situation changes substantially if the 1-dimensional string is replaced by a 3-dimensional string and the quantum tunneling turned into gluon splitting into quark-antiquark pair with a negligible momentum in the rest frame of the string. Fragmentation of such a string generates intrinsic transverse momenta which depends on the folding of the string and implies azimuthal correlations between hadrons. On the basis of angular properties of gluon emission under helicity conservation, it has been shown that the shape of the QCD string should be helix-like [2].

The helix-like shaped QCD string (or any 3-dimensional string) allows to develop fragmentation model where cross-talk between breakup vertices is possible (their distance is time-like). When the cross-talk (i.e. causal relation) between breakup vertices is imposed, the transverse shape of the string generates both transverse momentum and mass of the hadron. Quantization of the model then opens the way to build-up of hadron mass spectrum. It turns out that the causal constraint applied to the helical QCD field reveals a rather simple quantization pattern for particles with mass below $1 \mathrm{GeV}$. In particular, the pseudoscalar mesons $\left(\pi, \eta, \eta^{\prime}\right)$ can be regarded as string pieces fragmenting into $(\mathrm{n}=1,3,5)$ ground state hadrons (pions), with transverse mass $\left(E_{T}\right)$ and momentum $\left(p_{T}\right)$ of mesons defined by helical string properties [3]

$$
\begin{aligned}
E_{T} & =\sqrt{m_{n}^{2}+p_{T}^{2}}=n \kappa R \Delta \Phi, \\
p_{T} & =2 \kappa R|\sin (n \Delta \Phi / 2)|,
\end{aligned}
$$

\footnotetext{
a e-mail: sarka.todorova@cern.ch
} 
where $R$ stands for the radius of the helix, $\kappa \sim 1 \mathrm{GeV} / \mathrm{fm}$ is the string tension, $\Delta \Phi$ is the quantized helix phase difference describing the minimal piece of string which can form a hadron, and $m_{n}$ is the (quantized) meson mass spectrum.

The mass spectrum of pseudoscalar mesons is used to extract the parameters of the helical QCD field and its quantum properties; the fit of the spectrum indicates a rather narrow radius of the helical string $(\kappa R=68 \pm 2 \mathrm{MeV})$ and the quantized phase difference $\Delta \Phi=2.82 \pm 0.06 \mathrm{rad}$ which translates into a quantized ground state transverse energy $\left(\left.E_{T}\right|_{n=1} \sim 0.193 \mathrm{GeV}\right)$. It is the numerical value of $\Delta \Phi$ which suggests there should be a significant charge-combination asymmetry in the production of chains of ground state pions: while adjacent (unlike-sign) pairs of pions have to go apart in the transverse plane, the like-sign pion pairs with rank $2^{1}$ should have a relatively small opening angle of 2. $(\pi-2.8) \sim 0.7 \mathrm{rad}$. Charge-combination asymmetry in the production of pairs of charged pions thus may be understood as a consequence of local charge conservation in (coherent) QCD string fragmentation into a quantized chain of ground state pions.

It is an interesting observation that the $\kappa R$ obtained from the fit of the mass spectrum of pseudoscalar mesons agrees with the estimate of the size of the QCD flux tube derived from the fit of mass spectrum of glueball states using topological constants of knotted flux tubes [4].

Neglecting the longitudinal momentum differences between adjacent hadrons (i.e. assuming local homogenity of the fragmenting QCD field ), the momentum difference between ground state pions can be written as function of their rank $\mathrm{r}$ :

$$
Q(\mathrm{r})=\sqrt{-\left(p_{i}-p_{i+r}\right)^{2}} \cong 2 p_{T}^{t h r}|\sin (\mathrm{r} \Delta \Phi / 2)|,
$$

where $p_{T}^{\text {thr }}(\sim 134 \mathrm{MeV})$ stands for the intrinsic $p_{T}$ of ground state pions.

The numerical values of the predicted momentum difference separating pairs of ground state hadrons with rank up to 5 are given in Table 1.

The chain of $\mathrm{n}$ adjacent ground state pions has the mass

$$
M_{n h}=\sqrt{n^{2} m_{\pi}^{2}+\sum_{i \neq j} Q_{i j}^{2}}
$$

where $m_{\pi}$ is the pion mass, and $Q_{i j}$ the momentum difference between pairs of hadrons forming the chain.

\begin{tabular}{|c||c|c|c|c|c|}
\hline Pair rank difference & 1 & 2 & 3 & 4 & 5 \\
\hline \hline Q expected $[\mathrm{MeV}]$ & $266 \pm 8$ & $91 \pm 3$ & $236 \pm 7$ & $171 \pm 5$ & $178 \pm 5$ \\
\hline
\end{tabular}

Table 1. The expected momentum difference between ground state direct hadrons, in the quantized helix string model. The uncertainty $(3 \%)$ is derived from the precision of the fit of the mass spectrum of light pseudoscalar mesons[3].

\section{Coherent particle production}

2-particle correlations are usually studied as collective, incoherent effects. Experimentally, one tries to compare the measured inclusive 2-particle spectra with an 'uncorrelated' reference inclusive spectra,

\footnotetext{
${ }^{1}$ The rank of direct particles is equivalent to their ordering along the string : rank 0 is attributed to the decay products of a direct hadron, rank 1 designs adjacent hadrons, rank 2 hadrons separated by a common adjacent hadron et cetera; rank -1 is used to classify pairs of hadrons originating from different string/colour singlets. The decay products inherit the rank from their direct mother.
} 
where 'uncorrelated' means containing all dynamical correlations except those being measured. A well known example of this type of measurements is the study of Bose-Einstein effect, which is supposed to describe the excess of like-sign pairs of charged hadrons observed in all hadronic data samples. Since the Bose-Einstein symmetrization does not concern the pairs of hadrons with opposite charge, the inclusive spectrum of unlike-sign pairs can be used as a reference sample. The notion of the collective effect influences strongly the choice of the correlation function as ratio of the observed and the reference distribution. Assuming particle correlations arise in the hadronization process and
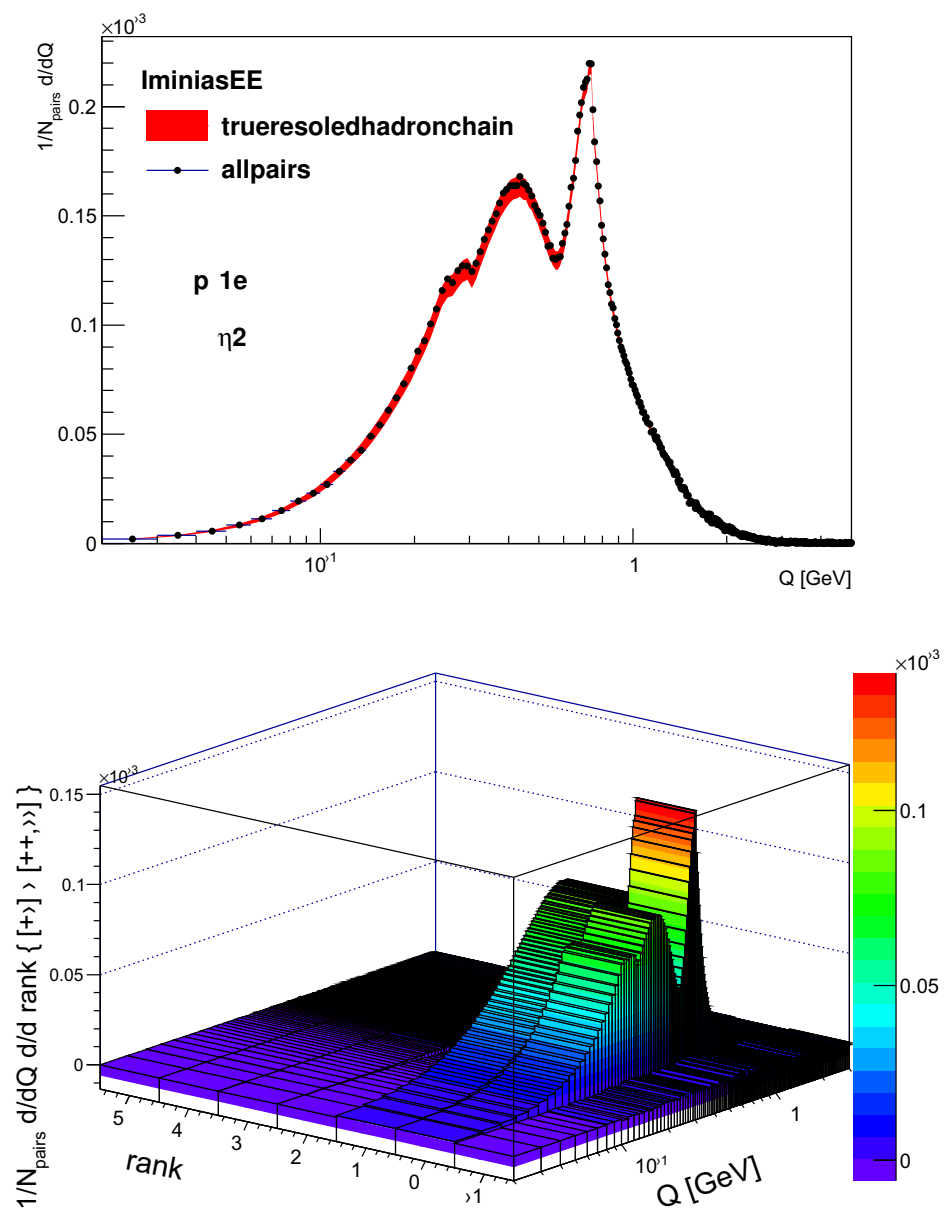

Figure 1. Upper plot: Comparison of the $\mathrm{Q}$ spectrum obtained by the means of subtraction of like-sign pair distribution from that of unlike-sign pairs, with the true $\mathrm{Q}$ spectrum for adjacent charged hadrons, obtained from MC truth (the uncertainty marked by red band reflects the uncertainty in the resolving the ordering within a decay of a direct hadron). Bottom plot: Detailed view of the rank dependence of the subtraction between unlike-sign and like-sign pairs in MC; pairs of hadrons originating from different strings (color-disconnected chains) are assigned rank=-1. Rank 0 designs pairs of hadrons from the decay of a direct common resonance. PYTHIA8[5] minimum bias sample for pp collisions at $\sqrt{s}=7 \mathrm{TeV}$ c.m.s.energy. 
reflect dynamical properties of the QCD field, the charge-combination asymmetry in the production of particles should be limited to adjacent hadrons ( bound by local charge conservation in the breakup of QCD field ), which can never form a like-sign pair. However, due to the (presumably random) presence of neutral hadrons in the QCD string fragmentation, it is unlikely that the charge-combination asymmetry holds for pairs with rank above 2 . If this is the case, the number of correlated hadron pairs should grow linearly with the number of charged hadrons (i.e. with the string length). Since the combinatorial background rises quadratically with the charged multiplicity, it represents a huge challenge, unless it is recognized that it can be completely eliminated by subtraction of like-sign and unlike-sign inclusive spectra. Thus it seems reasonable, for the study of adjacent hadron spectra, to replace the ratio of $\mathrm{Q}$ distributions by their difference. Indeed, the Monte-Carlo studies confirm the extraordinary power of the subtraction technique as illustrated by Fig. 1 - the level of precision with which the true shape of momentum difference for adjacent charged hadrons can be reproduced is within limits of the uncertainty in the resolving the order of the daughter hadrons in a multibody decay of a heavy resonance.

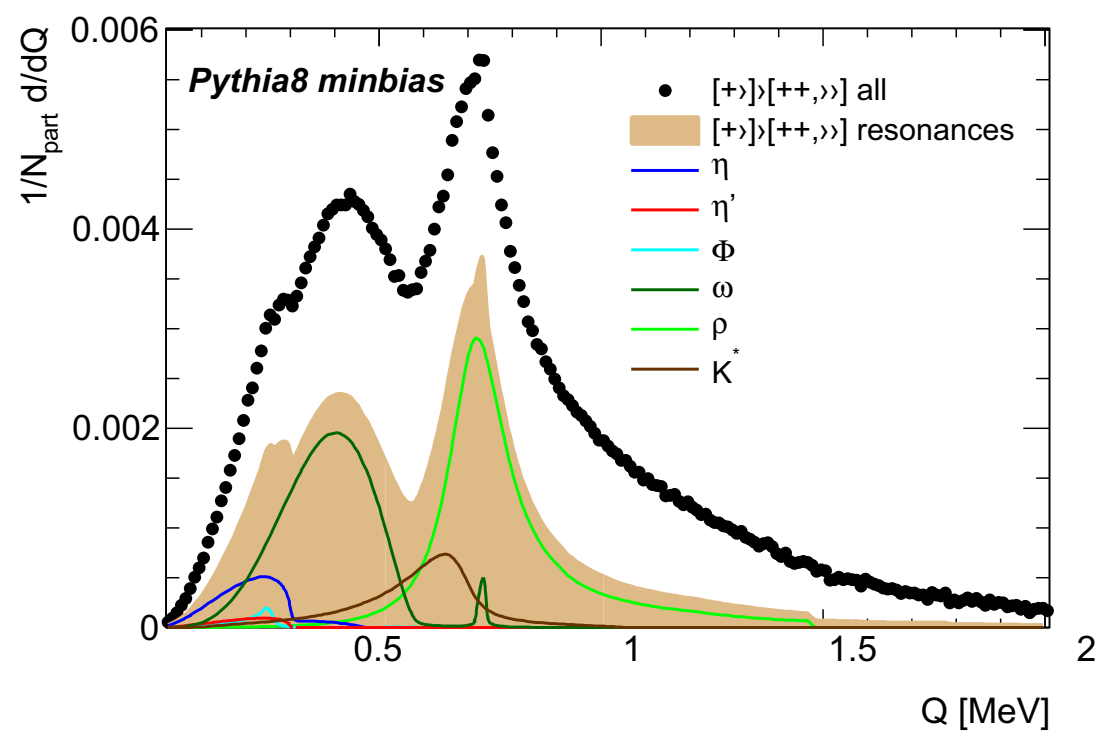

Figure 2. The Q spectrum generated by Pythia8 and the decomposition of its resonant part into leading contributions. The peak with lowest mass is formed by decay products of pseudoscalar mesons $\left(\eta, \eta^{\prime}\right)$ and the 2-body decay of the $\Phi$ resonance.

The shape of the subtracted distribution is expected to be driven by the resonance decays (Fig. 2). The dominant contribution comes from $\left[\eta, \eta^{\prime}, \Phi\right],[\omega]$ and $\left[\rho, K^{* 0}\right]$, which combine in 3 broad peaks. Please note that definition of primary particles ${ }^{2}$ does not include decay products of $\mathrm{K}$ mesons which therefore do not appear on the plot ( $K$ mesons are treated as stable particles).

\footnotetext{
${ }^{2}$ Primary particles are defined as all particles with lifetime longer than $0.3 \times 10^{-10}$ s originating from the primary interaction or from subsequent decay of particles with shorter lifetime.
} 


\section{Experimental data}

The predictions of the quantized fragmentation model can be verified using the Q distributions published by ATLAS[6]. The subtracted quantity

$$
\Delta Q\left(=N^{+-}(Q)-N^{++,--}(Q)\right)
$$

is fitted - in the region up to $\mathrm{Q}=0.4 \mathrm{GeV}$ - by 3 gaussians describing the enhanced production of pairs of like-sign hadrons (seen as depletion at low Q), and the first 2 (positive) peaks for adjacent unlikesign pairs of hadrons. The shape of the enhanced production of like-sign pairs is in good agreement with the model prediction for the size of momentum difference between rank 2 hadrons in the chain of ground state hadrons (pions), with the maximum (turned minimum) at $\mathrm{Q}=0.09 \mathrm{GeV}$. The position of the lowest peak for unlike-sign pairs agrees with the model prediction for the minimal momentum difference between a pair of adjacent ground state hadrons $(0.26 \mathrm{GeV})$.

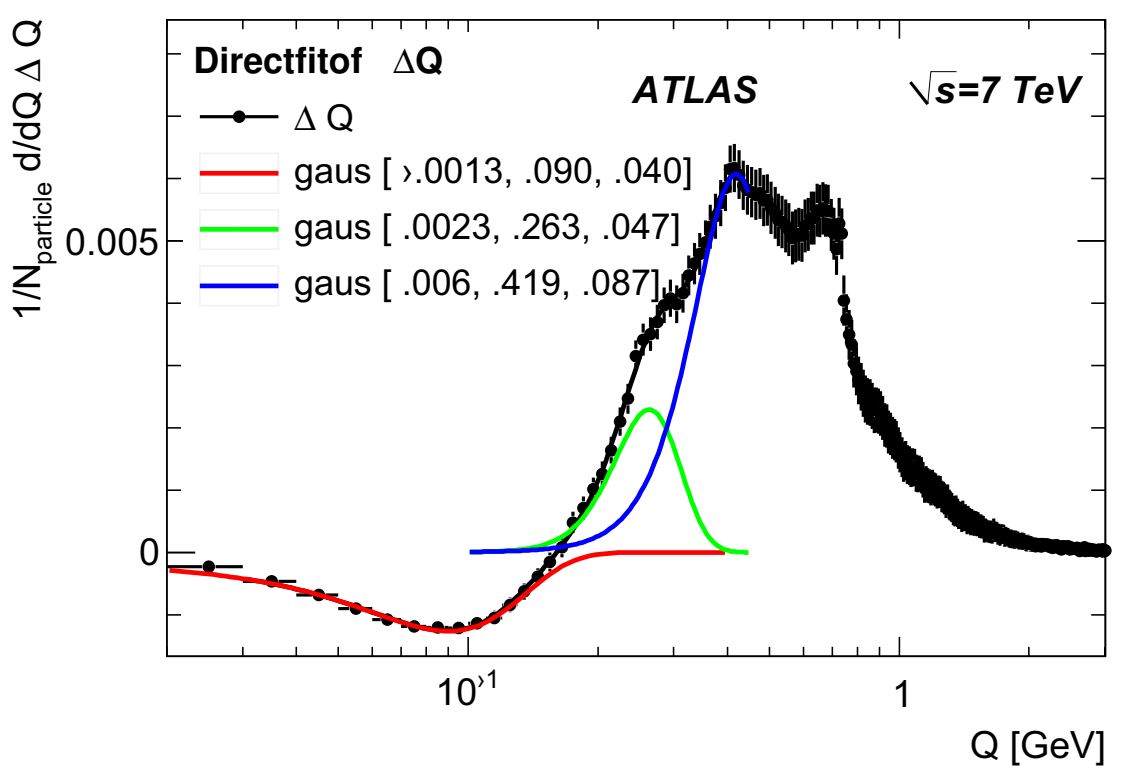

Figure 3. Direct fit of the triple gaussian shape at low $\mathrm{Q}$ in the inclusive $\Delta Q$ distribution. The onset of adjacent pair production coincides with the position of (the first) $Q^{+-}$peak at $\mathrm{Q}=0.26 \mathrm{GeV}$. The ATLAS data are taken from Ref [6].

\section{Conclusion}

The combination of causal and quantum constraints allows to develop model of QCD string fragmentation where the shape of QCD field translates into observable properties (mass, transverse momentum) of hadrons. Parameters of the QCD string are fitted using mass spectrum of pseudoscalar mesons. Due to the reduction of the number of free parameters, the model acquires considerable predictive power. The measured shape of 2-particle correlations (commonly attributed to Bose-Einstein effect) agrees with model predictions, which means the effect (i.e.enhanced production of pairs of like-signed charged hadrons ) can be attributed to the coherent particle production. 


\section{References}

[1] B. Andersson, et al. Parton Fragmentation and String Dynamics, Phys. Rept. 97 (1983) 31.

[2] B. Andersson, G. Gustafson, J. Hakkinen, M. Ringner and P. Sutton, Is there screwiness at the end of the QCD cascades?, JHEP 9809 (1998) 014.

[3] Š. Todorova, Quantization of the QCD string with a helical structure, Phys.Rev.D89, 015002 (2014).

[4] R.V.Buniy, et al.,The tight knot spectrum in QCD, Phys.Rev.D89, 054513 (2014).

[5] T. Sjostrand, S. Mrenna and P. Z. Skands, Comput. Phys. Comm. 178 (2008)

[6] ATLAS Collaboration, Eur.Phys.J.C75 (2015) 466. 